\title{
Pulse-shape determination of intracavity compressed picosecond pulses by two-photon fluorescence analysis
}

\author{
P. SPERBER, A. PENZKOFER \\ Naturwissenschaftliche Fakultät II - Physik, Universität Regensburg, 8400 \\ Regensburg, FRG
}

Received 2 December 1985; revised 29 January 1986

Intracavity pulse compression of self-phase modulated pulses in passively modelocked lasers leads to temporally structured pulses. Experimental two-photon fluorescence traces are decorrelated to determine the approximate temporal shape of the modulated pulses.

\section{Introduction}

The two-photon fluorescence technique is widely used to determine the duration of smooth picosecond light pulses [1-3]. In recent experiments self-phase modulated picosecond pulses in modelocked lasers have been compressed by the finite gain width of the active medium [4-8]. These intracavity compressed pulses consist of a short spike upon a broad pedestal. The pedestal may be reduced by the nonlinear transmission through saturable absorbers.

In this paper the shape of composed and modulated pulses is reconstructed from two-photon fluorescence traces. First, a short derivation of the two-photon fluorescence equations is given. The proportionality constant between fluorescence signal and autocorrelation function is explicitly determined. Then, two-photon fluorescence traces for various pulse shapes are calculated. The results are used to determine the approximate temporal shape of intracavity compressed pulses by decorrelating experimental two-photon fluorescence traces.

\section{Theory}

In a two-photon fluorescence arrangement the laser pulses are split into two parts that cross in a cell containing a two-photon absorbing and single-photon fluorescing dye. A typical colliding pulse two-photon fluorescence arrangement is shown in Fig. 1a. A level scheme of the dye is depicted in Fig. 1b (no single-photon absorption present). The fluorescence is enhanced in the region of temporal overlap of the colliding pulses [1-3]. The intensity dissipation of two-photon absorption is given by [9]

$$
\frac{\partial I}{\partial z}=-\left\langle\boldsymbol{E} \frac{\partial \boldsymbol{P}_{\mathrm{TPA}}}{\partial t}\right\rangle
$$

where $I$ is the intensity of the light pulse, $\boldsymbol{E}$ is its electrical field strength, $\boldsymbol{P}_{\text {TPA }}$ is the nonlinear polarization responsible for two-photon absorption and $z$ is the spatial coordinate of light propagation. The brackets $\langle>$ symbolize the temporal average over a period of $2 \pi / \omega(\omega$ is the angular laser frequency). In the quasi-monochromatic plane-wave approximation of linear polarized light $\boldsymbol{E}$ and $\boldsymbol{P}_{\mathrm{TPA}}$ are given by [10]

$$
\begin{aligned}
\boldsymbol{E} & =\frac{1}{2}\left[E_{0} \exp (\mathrm{i} \omega t)+\text { c.c. }\right] \boldsymbol{e}_{\mathrm{x}} \\
\boldsymbol{P}_{\mathrm{TPA}} & =\frac{1}{2}\left[P_{\mathrm{TPA}} \exp (\mathrm{i} \omega t)+\text { c.c. }\right] \boldsymbol{e}_{\mathrm{x}}
\end{aligned}
$$




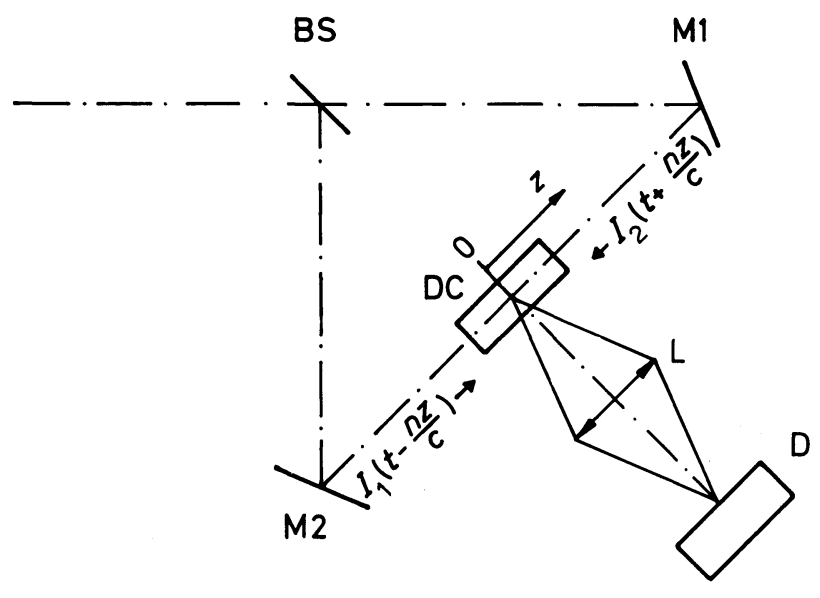

(a)

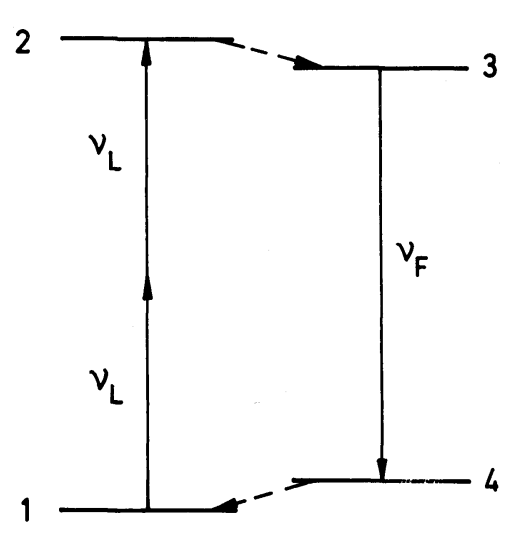

(b)

Figure 1 (a) Two-photon fluorescence arrangement and (b) level system of two-photon fluorescing dye: BS, 50\%beam splitter; M1, M2, 100\% mirrors; DC, dye cell; L, imaging optics; D, detection system (SIT-vidicon); $v_{L}$, laser frequency; $v_{F}$, fluorescence frequency.

where $P_{\mathrm{TPA}}=3 \varepsilon_{0} \chi_{\mathrm{xxxx}}^{(3)}(-\omega ; \omega,-\omega, \omega)\left|E_{0}\right|^{2} E_{0}$. Insertion of Equations 2 and 3 into Equation 1 and using the slowly varying amplitude approximation gives

$$
\frac{\partial I}{\partial z}=-\frac{3}{2} \varepsilon_{0} \omega \chi_{\mathrm{xxxx}}^{(3)^{\prime \prime}}(-\omega ; \omega,-\omega, \omega)\left|E_{0}\right|^{4}
$$

Only the imaginary part of the nonlinear susceptibility $\chi^{(3)}=\chi^{(3)^{\prime}}-\mathrm{i} \chi^{(3)^{\prime \prime}}$ remains in Equation 4. For a single pulse the intensity $I$ is related to the electric-field amplitude by $I=\left(\varepsilon_{0} c n / 2\right)\left|E_{0}\right|^{2}$ and Equation 4 reduces to

$$
\frac{\partial I}{\partial z}=-\alpha^{(2)} I^{2}
$$

with

$$
\alpha^{(2)}=\frac{6 \omega}{c^{2} n^{2} \varepsilon_{0}} \chi_{\mathrm{xxxx}}^{(3)^{\prime \prime}}(-\omega ; \omega,-\omega, \omega)
$$

where $\alpha^{(2)}=N \sigma^{(2)} /(\hbar \omega)$ (with $\mathrm{cm} \mathrm{W}^{-1}$ ) is the two-photon absorption coefficient; $\sigma^{(2)}$ is the twophoton absorption cross-section (units $\mathrm{cm}^{4} \mathrm{~s}$ ) and $N$ is the number density of two-photon absorbing dye molecules.

In the colliding pulse two-photon fluorescence arrangement the amplitude of the electrical field strength $E_{0}$ is given by $\left(E_{01}\right.$ and $E_{02}$ real)

$$
E_{0}(\boldsymbol{r}, t)=E_{01}(x, y, t-z n / c) \exp \left(-\mathrm{i} k z+i \phi_{1}\right)+E_{02}(x, y, t+z n / c) \exp \left(\mathrm{i} k z+\mathrm{i} \phi_{2}\right)
$$

Beam focusing or defocusing is excluded. Otherwise $E_{01}$ and $E_{02}$ would depend explicitly on $z$. Equation 4 yields, by insertion of Equation $7\left(I_{1}=I_{1}(x, y, t-z n / c), I_{2}=I_{2}(x, y, t+z n / c)\right)$

$$
\begin{aligned}
\frac{\partial I}{\partial z}= & -\alpha^{(2)}\left\{I_{1}^{2}+I_{2}^{2}+4 I_{1} I_{2}+2 I_{1} I_{2} \cos \left[4 k z+2\left(\phi_{2}-\phi_{1}\right)\right]\right. \\
& \left.+4\left(I_{1} I_{2}\right)^{1 / 2}\left(I_{1}+I_{2}\right) \cos \left(2 k z+\phi_{2}-\phi_{1}\right)\right\}
\end{aligned}
$$

The spatial modulation with $\pi / k=\lambda / 2$ and $\pi / 2 k=\lambda / 4$ is only observed in high resolution collinear 
propagation experiments $[11,12]$ (resolution element of vidicon is $25 \mu \mathrm{m} \gg \lambda$ ). Neglecting the unresolved modulation, Equation 8 reduces to

$$
\frac{\partial I}{\partial z}=-\alpha^{(2)}\left[I_{1}^{2}\left(x, y, t-\frac{n z}{c}\right)+I_{2}^{2}\left(x, y, t+\frac{n z}{c}\right)+4 I_{1}\left(x, y, t-\frac{n z}{c}\right) I_{2}\left(x, y, t+\frac{n z}{c}\right)\right]
$$

The time-integrated fluorescence density, $\varepsilon_{\mathrm{F}}$, at position $\boldsymbol{r}^{\prime}=(x, y, z)\left(\right.$ units $\left.\mathrm{J} \mathrm{cm}^{-3}\right)$ is obtained by time integration over the intensity dissipation at position $\boldsymbol{r}$

$$
\begin{aligned}
\varepsilon_{\mathrm{F}}(x, y, z)= & -q_{\mathrm{F}} \frac{\omega_{\mathrm{F}}}{2 \omega} \int_{-\infty}^{\infty} \frac{\partial I}{\partial z} \mathrm{~d} t \\
= & q_{\mathrm{F}} \frac{\omega_{\mathrm{F}}}{2 \omega} \alpha^{(2:}\left[\int_{-\infty}^{\infty} I_{1}^{2}\left(x, y, t^{\prime}\right) \mathrm{d} t^{\prime}+\int_{-\infty}^{\infty} I_{2}^{2}\left(x, y, t^{\prime}\right) \mathrm{d} t^{\prime}\right] \\
& \times\left\{1+4 \frac{\int_{-\infty}^{\infty} I_{1}\left(t^{\prime}\right) I_{2}\left(t^{\prime}+\frac{2 n z}{c}\right) \mathrm{d} t^{\prime}}{\int_{-\infty}^{\infty} I_{1}^{2}\left(t^{\prime}\right) \mathrm{d} t^{\prime}+\int_{-\infty}^{\infty} I_{2}^{2}\left(t^{\prime}\right) \mathrm{d} t^{\prime}}\right\}
\end{aligned}
$$

where $q_{\mathrm{F}}$ is the fluorescence quantum yield, and $\omega_{\mathrm{F}}$ is the fluorescence frequency. The total fluorescence signal $S$ at position $z$ is found by integration over the cross-sectional coordinates $x$ and $y$ (units $\mathrm{J} \mathrm{cm}^{-1}$ )

$$
\begin{aligned}
S(z)= & q_{\mathrm{F}} \frac{\omega_{\mathrm{F}}}{2 \omega} \alpha^{(2)}\left[\int \mathrm{d} x \int \mathrm{d} y s_{1}^{2}(x, y) \int_{-\infty}^{\infty} I_{10}^{2}\left(t^{\prime}\right) \mathrm{d} t^{\prime}+\int \mathrm{d} x \int \mathrm{d} y s_{2}^{2}(x, y) \int_{-\infty}^{\infty} I_{20}^{2}\left(t^{\prime}+\frac{2 n z}{c}\right) \mathrm{d} t^{\prime}\right] \\
& \times\left\{1+4 \frac{\int \mathrm{d} x \int \mathrm{d} y s_{1}(x, y) s_{2}(x, y) \int_{-\infty}^{\infty} I_{10}\left(t^{\prime}\right) I_{20}\left(t^{\prime}+\frac{2 n z}{c}\right) \mathrm{d} t^{\prime}}{\int \mathrm{d} x \int \mathrm{d} y s_{1}^{2}(x, y) \int_{-\infty}^{\infty} I_{10}^{2}\left(t^{\prime}\right) \mathrm{d} t^{\prime}+\int \mathrm{d} x \int \mathrm{d} y s_{2}^{2}(x, y) \int_{-\infty}^{\infty} I_{20}^{2}\left(t^{\prime}\right) \mathrm{d} t^{\prime}}\right\}
\end{aligned}
$$

where $I_{1}\left(x, y, t^{\prime}\right)=s_{1}(x, y) I_{10}\left(t^{\prime}\right)$ and $I_{2}\left(x, y, t^{\prime}\right)=s_{2}(x, y) I_{20}\left(t^{\prime}\right)$ has been used in Equation 11 .

In the following, only the special case of $s_{1}(x, y)=s_{2}(x, y)=s(x, y)$ (same spatial shape and perfect beam overlap) and $I_{10}=I_{20}=I_{0}(50 \%$ beam splitter in two-photon fluorescence arrangement, no pulse focusing) is discussed. Equation 11 reduces to

$$
S(z)=q_{\mathrm{F}} \frac{\omega_{\mathrm{F}}}{\omega} \alpha^{(2)} A[1+2 G(\tau)]
$$

with

$$
\begin{gathered}
A=\int \mathrm{d} x \int \mathrm{d} y s^{2}(x, y) \int_{-\infty}^{\infty} I_{0}^{2}\left(t^{\prime}\right) \mathrm{d} t^{\prime} \\
G(\tau)=\frac{\int_{-\infty}^{\infty} I_{0}\left(t^{\prime}\right) I_{0}\left(t^{\prime}+\tau\right) \mathrm{d} t^{\prime}}{\int I_{0}^{2}\left(t^{\prime}\right) \mathrm{d} t^{\prime}} \\
\tau=2 n z / c
\end{gathered}
$$

and $n$ is the refractive index of the two-photon absorbing dye solution. Equation 12 gives the fluorescence signal as a function of the fluorescence quantum efficiency and the two-photon absorption coefficient of the two-photon absorbing dye. It may be used for appropriate dye selection for two-photon fluorescence measurements.

For pulsed emission the second-order autocorrelation function $G(\tau)$ gives $G(0)=1$ and $G( \pm \infty)=0$ resulting in a contrast ratio of $S(0) / S(\infty)=3$ (only valid for the special case of $I_{10}=I_{20}=I_{0}$ ). The half-width (FWHM) of the autocorrelation function $\Delta t$ is related to the 
halfwidth (FWHM) of the pulse $\Delta t$ by $\Delta \tau=\gamma \Delta t$. The ratio $\gamma$ depends on the pulse shape. It is $\gamma=2^{1 / 2}$ for gaussian shape, $\gamma=1$ for rectangular shape and $\gamma=2$ for lorentzian shape. $G(\tau)$ is a symmetric function $[G(\tau)=G(-\tau)]$ and therefore does not reveal pulse asymmetry.

In the following we analyse two-photon fluorescence traces of (i) two combined gaussian pulses of $1 / \mathrm{e}$ widths, $t_{\mathrm{s}}$ (short) and $t_{1}$ (long), centred at the same position with relative heights $r_{\mathrm{s}}$ and $r_{1}\left(r_{\mathrm{s}}+r_{1}=1\right)$

$$
I_{0}\left(t^{\prime}\right)=I_{0}\left[r_{\mathrm{s}} \exp \left(-t^{2} / t_{\mathrm{s}}^{2}\right)+r_{1} \exp \left(-t^{2} / t_{1}^{2}\right)\right]
$$

(ii) two combined pulses with different centre position

$$
I_{0}\left(t^{\prime}\right)=I_{0}\left\{r_{\mathrm{s}} \exp \left(-t^{\prime 2} / t_{\mathrm{s}}^{2}\right)+r_{1} \exp \left[-\left(t^{\prime}-t_{\mathrm{p}}\right)^{2} / t_{1}^{2}\right]\right\}
$$

(iii) a central pulse with two side peaks $\left(r_{\mathrm{s}}+r_{11}+r_{12}=1\right)$

$I_{0}\left(t^{\prime}\right)=I_{0}\left\{r_{\mathrm{s}} \exp \left(-t^{2} / t_{\mathrm{s}}^{2}\right)+r_{11} \exp \left[-\left(t^{\prime}-t_{\mathrm{p} 1}\right)^{2} / t_{11}^{2}\right]+r_{12} \exp \left[-\left(t^{\prime}+t_{\mathrm{p} 2}\right)^{2} / t_{12}^{2}\right]\right\}$

(iv) a sine-modulated gaussian pulse

$$
I_{0}\left(t^{\prime}\right)=I_{0} \frac{1}{1+m}\left[1+m \cos \left(\frac{\pi}{2} \frac{t^{\prime}}{t_{\mathrm{m}}}+\phi\right)\right] \exp \left(-t^{2} / t_{0}^{2}\right)
$$

In the analysis $I_{0}\left(t^{\prime}\right)$ of Equations 16 to 19 is entered into Equation 14 and the fluorescence traces are calculated according to Equation 12.

In Equations 16 to 19 pulse attenuation due to single-photon absorption, light scattering or two-photon absorption is neglected. Otherwise $I_{0}$ would depend on propagation distance $z$. This situation is analysed in [13]. In our experimental studies, single-photon absorption and light scattering is undetectably small for the dye solution of $2.5 \times 10^{-3} \mathrm{M}$ rhodamine $6 \mathrm{G}$ in ethanol (sample length $l=2 \mathrm{~cm}$ for ruby laser; $l=1 \mathrm{~cm}$ for Nd-glass laser). Measurable pulse attenuation by two-photon absorption [13] or by other nonlinear effects [14 to 17] does not occur because the pulse intensity in the two-photon fluorescing dye cell is kept at moderate values $\left(I_{0}<10^{9} \mathrm{~W} \mathrm{~cm}^{-2}\right)$. Two-photon fluorescence detection at low pulse intensities is achieved by large-aperture collecting optics and sensitive recording (SIT-vidicon). The two-photon absorption is responsible for the observed fluorescence, but it is so weak that it does not reduce measurably the pulse intensity. Perfect beam overlap $\left(s_{1}(x, y)=s_{2}(x, y)\right)$ and exact $50 \%$ beam splitting $\left(I_{10}=I_{20}\right)$ is more difficult to achieve. Deviation from this ideal situation reduces the contrast ratio $S(0) / S(\infty)$ to less than three but the temporal widths remain almost unchanged. In the decorrelation of the experimental two-photon fluorescence traces (Section 4), traces with small deviation from contrast ratio three are normalized according to $S(\tau) / S(\infty)=1+2\left[S_{\mathrm{m}}(\tau)-S_{\mathrm{m}}(\infty)\right] /\left[S_{\mathrm{m}}(0)-S_{\mathrm{m}}(\infty)\right]$, where $S_{\mathrm{m}}(\tau)$, $S_{\mathrm{m}}(0)$ and $S_{\mathrm{m}}(\infty)$ are the experimental fluorescence signals.

\section{Two-photon fluorescence traces of structured pulses}

Two gaussian pulses centred at the same position are analysed in Fig. 2a and a' (Equation 16). The curves indicate that a short pulse on a broad pedestal shows up in a small spike upon a broad two-photon fluorescence trace. The half-widths of the short fluorescence spike and of the broad fluorescence peak agree reasonably well with the half-widths of the fluorescence traces of the corresponding single pulses $\left(\gamma \approx 2^{1 / 2}\right.$ for both pulses). This fact allows the separate measurement of the pulse durations $\Delta t_{\mathrm{s}}$ and $\Delta t_{1}$ of the composed pulses. The peak fluorescence height $S_{1}(0)$ of the broad pulse is determined by the ratio of the pulse heights $r_{\mathrm{s}} / r_{1}$ and the ratio of the pulse durations $\Delta t_{\mathrm{s}} / \Delta t_{1}$. The solid curves in Fig. 3a depict $S_{1}(0) / S(\infty)$ against $r_{\mathrm{s}}$ for two pulse duration ratios $\left(\Delta t_{\mathrm{s}} / \Delta t_{1}=0.1\right.$ and 0.025$)$. The corresponding curves of $S_{1}(0) / S(\infty)$ against the ratio $W_{\mathrm{S}} / W_{0}$ of short pulse-energy content $W_{\mathrm{S}}$ to combined pulse-energy content $W_{0}$ are shown in Fig. 3b. If both pulses have the same peak intensity $\left(r_{\mathrm{s}}=0.5\right)$ and the pulse duration ratio is $\Delta t_{\mathrm{s}} / \Delta t_{1}=0.1$, then the broad fluorescence peak is at $S_{1}(0) / S(\infty) \simeq 2$.9. If, on the other hand, both pulses have the same energy 


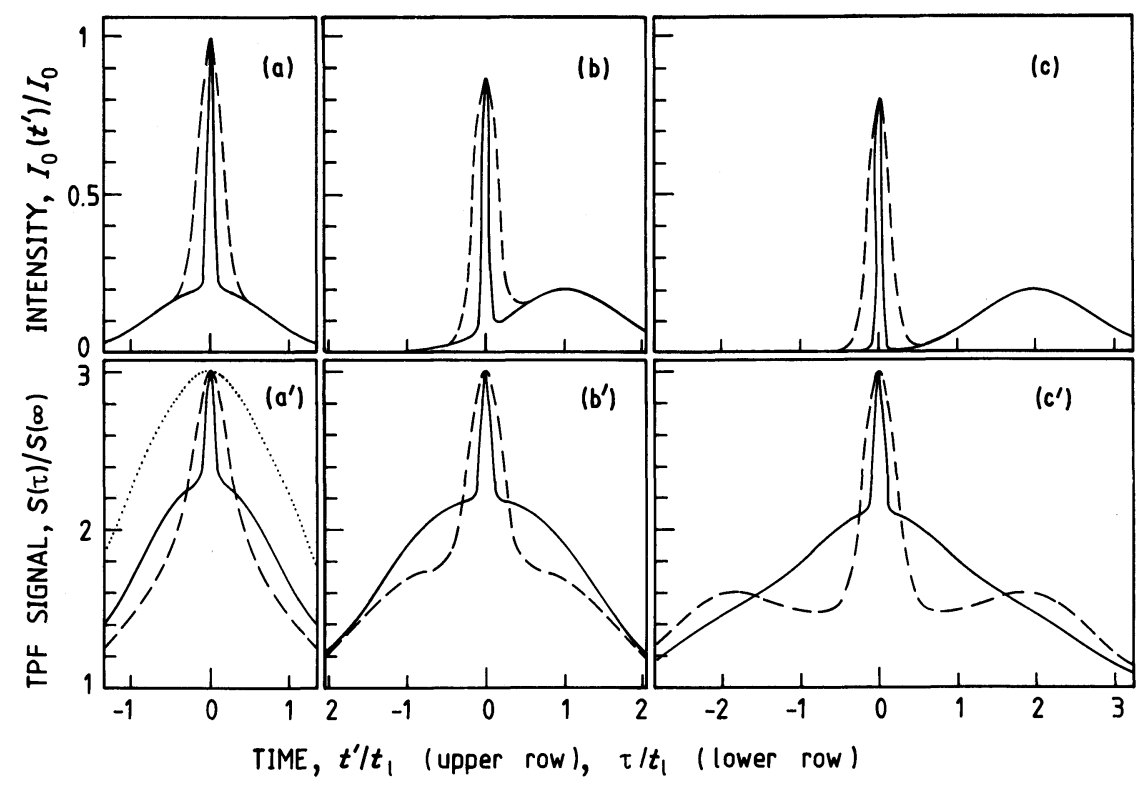

Figure 2 Pulse shapes and two-photon fluorescence traces of composed gaussian pulses: intensity ratio $r_{\mathrm{s}}=0.8$; time ratio $\Delta t_{\mathrm{s}} / \Delta t_{1}=0.05(-)$ and $0.2(---)$. Pulse separations $\left(a, a^{\prime}\right) t_{\mathrm{p}}=0 ;\left(b, b^{\prime}\right) t_{\mathrm{p}}=t_{1}$ and $\left(c, c^{\prime}\right) t_{\mathrm{p}}=2 t_{1}$. Dotted curve $(\cdots)$ in $\left(a^{\prime}\right)$ belongs to a single gaussian pulse of duration $\Delta t_{1}$.

content, then the broad pulse fluorescence peak is at $S_{1}(0) / S(\infty)=1.26$. In this case the high intensity of the short pulse dominates the two-photon fluorescence.

In Fig. $2 \mathrm{~b}$ and $\mathrm{c}$ combined gaussian pulses centred at different positions are shown (Equation 17). The corresponding two-photon fluorescence traces are displayed in Fig. $2 b^{\prime}$ and $c^{\prime}$. The short pulse shows up in a sharp spike at the centre of the fluorescence trace with a half-width of

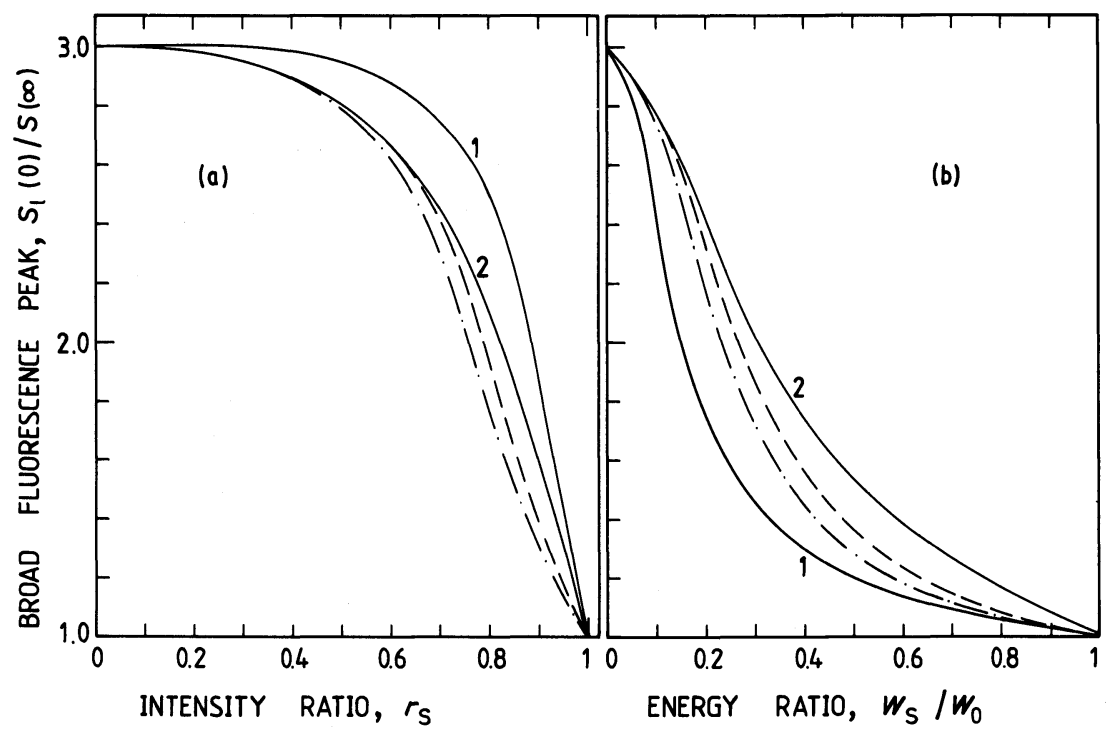

Figure 3 Contribution of broad pulse to two-photon fluorescence trace in the case of composed pulses (composition of two gaussian pulses): (a) dependence of broad fluorescence peak $S_{1}(0)$ on the short pulse intensity contribution $r_{\mathrm{s}}$; (b) $S_{1}(0)$ against energy ratio $W_{\mathrm{s}} / W_{0}$ of short pulse to combined pulse. (-) Centred pulses, $t_{\mathrm{p}}=0$, with $\Delta t_{\mathrm{s}} / \Delta t_{\mathrm{l}}=0.025$ (Curve 1) and $\Delta t_{\mathrm{s}} / \Delta t_{\mathrm{l}}=0.1$ (Curve 2). (---) $t_{\mathrm{p}}=t_{\mathrm{l}}$ and $\Delta t_{\mathrm{s}} / \Delta t_{1}=0.1$. (-.-.) $t_{\mathrm{p}}=2 t_{1}$ and $\Delta t_{\mathrm{s}} /$ $\Delta t_{1}=0.1$. 


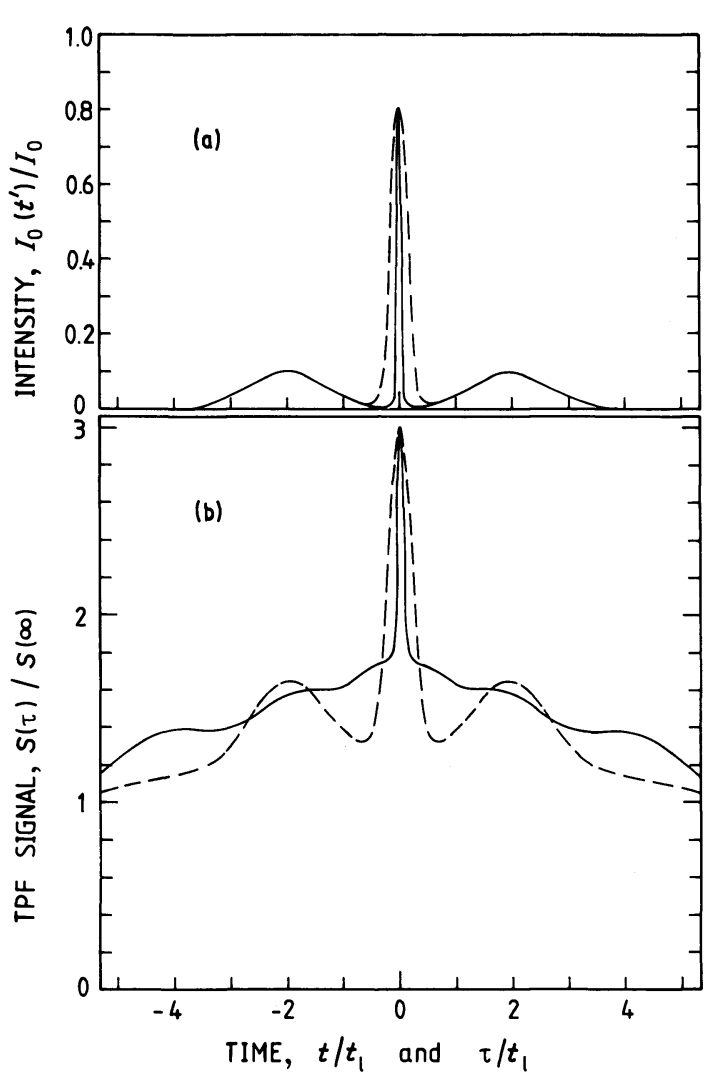

Figure 4 (a) Pulse shapes and (b) two-photon fluorescence traces of pulses with two symmetrical side peaks (composition of three gaussian pulses): (-) intensity ratio, $r_{\mathrm{s}}=0.8$, pulse width of short pulse $\Delta \tau_{\mathrm{s}}=0.05 \Delta t_{1}$; $(--) r_{\mathrm{s}}=0.8$ and $\Delta t_{\mathrm{s}}=0.2 \Delta t_{\mathrm{l}}$.

$\Delta \tau_{\mathrm{s}} \simeq 2^{1 / 2} \Delta t_{\mathrm{s}}$. The broad pulse causes a fluorescence trace of half-width $\Delta \tau_{1} \simeq 2^{1 / 2} \Delta t_{1}$ centred at $\tau=0$ by correlation with itself. The correlation of the broad pulse with the short pulse results in two fluorescence pulses of width $\left(\Delta t_{1}^{2}+\Delta t_{\mathrm{s}}^{2}\right)^{1 / 2}$ centred at $\tau=t_{\mathrm{p}}$ and $\tau=-t_{\mathrm{p}}$. As is seen in Fig. $2 \mathrm{~b}^{\prime}$ and $\mathrm{c}^{\prime}$ the broad fluorescence traces centred at $\tau=0$ and $\tau= \pm t_{\mathrm{p}}$ overlap and their separate shapes are not always resolved. The dependence of $S_{1}(0) / S(\infty)$ against $r_{\mathrm{s}}$ and $W_{\mathrm{s}} / W_{0}$ is depicted in Fig. $3 \mathrm{a}$ and $\mathrm{b}$, respectively, for $\Delta t_{\mathrm{s}} / \Delta t_{1}=0.1$. The behaviour is similar to the centred pulses.

The situation of a short central pulse with two symmetrical broad side pulses is illustrated in Fig. 4 (Equation 19 with $r_{11}=r_{12}=r_{1} / 2$ and $t_{\mathrm{p} 1}=t_{\mathrm{p} 2}=t_{\mathrm{p}}$ ). The fluorescence trace extends over a region of $\Delta \tau_{1}+4 t_{\mathrm{p}}\left(\Delta \tau_{1}=\gamma \Delta t_{1}\right)$. It is composed of a central spike of width $\Delta \tau_{\mathrm{s}}=2^{1 / 2} \Delta t_{\mathrm{s}}$, a broad central trace of width $\Delta \tau_{1}=2^{1 / 2} \Delta t_{1}$ (correlation of each side pulse with itself), two fluorescence spikes centred at $\tau= \pm t_{\mathrm{p}}$ of width $\left(\Delta t_{1}^{2}+\Delta t_{\mathrm{s}}^{2}\right)^{1 / 2}$ (correlation of each side pulse with central short pulse), and two fluorescence pulses centred at $\tau= \pm 2 t_{\mathrm{p}}$ and of width $2^{1 / 2} \Delta t_{1}$ (correlation of the two side pulses). Depending on the pulse duration ratio $\Delta t_{\mathrm{s}} / \Delta t_{1}$, the intensity ratio $r_{\mathrm{s}} / r_{1}$ and the pulse separation $t_{\mathrm{p}}$, the fluorescence components are more or less well resolved.

An irregular burst of pulses results in washing out the fringes on both sides of the central spike of the two-photon fluorescence trace [3, 18] (no pictures are shown here).

Two-photon fluorescence traces of sine-modulated pulses (Equation 19) are plotted in Fig. 5. The envelope width of the two-photon fluorescence trace is related to the envelope width of the modulated pulse by $\Delta \tau=\gamma \Delta t$ ( $\gamma$ unchanged, e.g. $\gamma=2^{1 / 2}$ for gaussian envelope). The modulation spacing $\tau_{\mathrm{m}}$ of the fluorescence trace is equal to the modulation spacing $t_{\mathrm{m}}$ of the pulse. The contrast of the fluorescence trace $m_{\mathrm{TPF}}=\left(S_{\max }-S_{\min }\right) /\left(S_{\max }+S_{\min }-2 S(\infty)\right)$ is smaller than the modulation depth $m=\left(I_{\max }-I_{\min }\right) /\left(I_{\max }+I_{\min }\right)$ of the pulse. A plot of $m_{\mathrm{TPF}}$ against $m$ is depicted in Fig. 6. The reduction in contrast is due to the temporal broadening caused by the correlation of the 


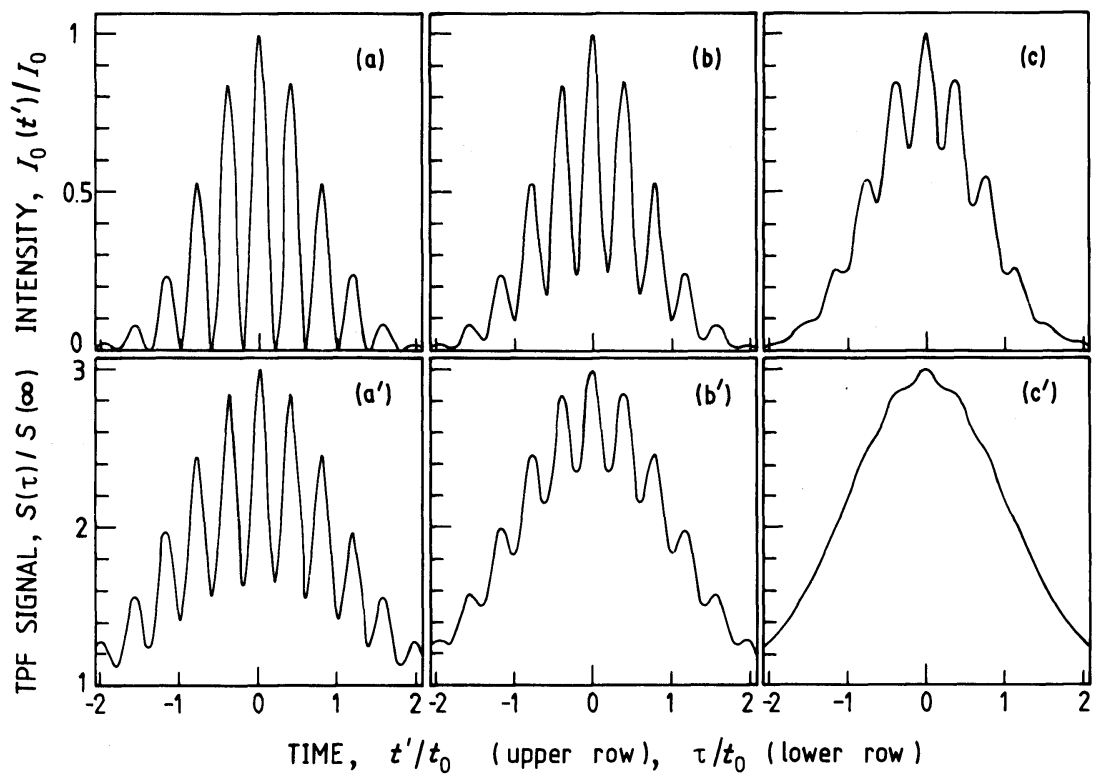

Figure 5 Pulse shapes and two-photon fluorescence traces of cosine-modulated pulses: $\left(a, a^{\prime}\right) m=\left(I_{\max }-I_{\min }\right) /$ $\left(I_{\max }+I_{\min }\right)=1 ;\left(\mathrm{b}, \mathrm{b}^{\prime}\right) m=0.6 ;\left(\mathrm{c}, \mathrm{c}^{\prime}\right) m=0.2$. Modulation spacing $t_{\mathrm{m}}=0.1 t_{0}$ (Equation 19).

pulses. The two-photon fluorescence traces are found to be independent of the phase $\phi$ of Equation $19\left(\phi=0\right.$ give a peak at $t^{\prime}=0 ; \phi=\pi / 2$ gives a minimum at $\left.t^{\prime}=0\right)$.

\section{Approximate decorrelation of experimental two-photon fluorescence traces}

In Figs 7 and 8 experimental two-photon fluorescence traces are shown (solid curves). The curves of Fig. 7 belong to a passively mode-locked ruby laser [5]. The pulses towards the end of the pulse train are self-phase modulated. The temporal pulse compression is caused by the finite spectral gain width of the active medium which cannot amplify the whole spectral width of the self-phase modulated pulses. The solid curve in Fig. 7a is obtained without an external saturable absorber; the solid curve in Fig. $7 \mathrm{~b}$ was obtained by passing the pulse through an external saturable absorber

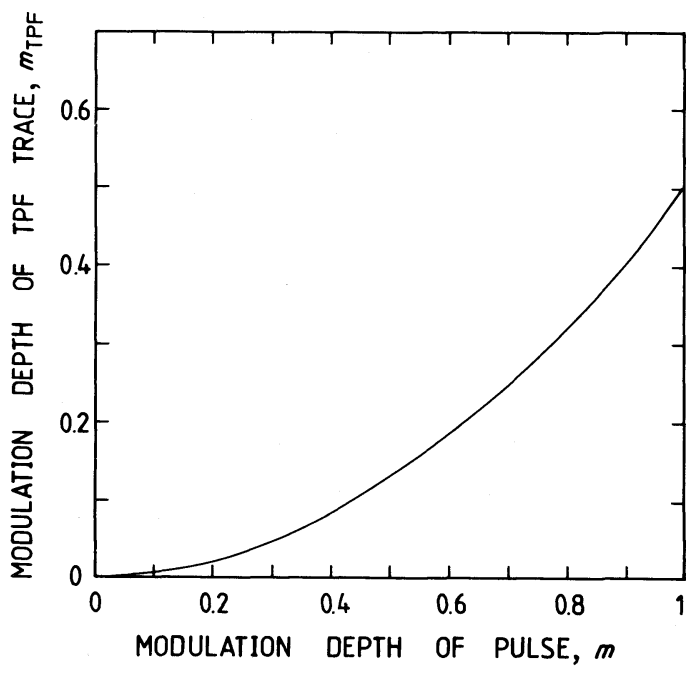

Figure 6 Fringe contrast of two-photon fluorescence traces as a function of modulation depth of pulses. 


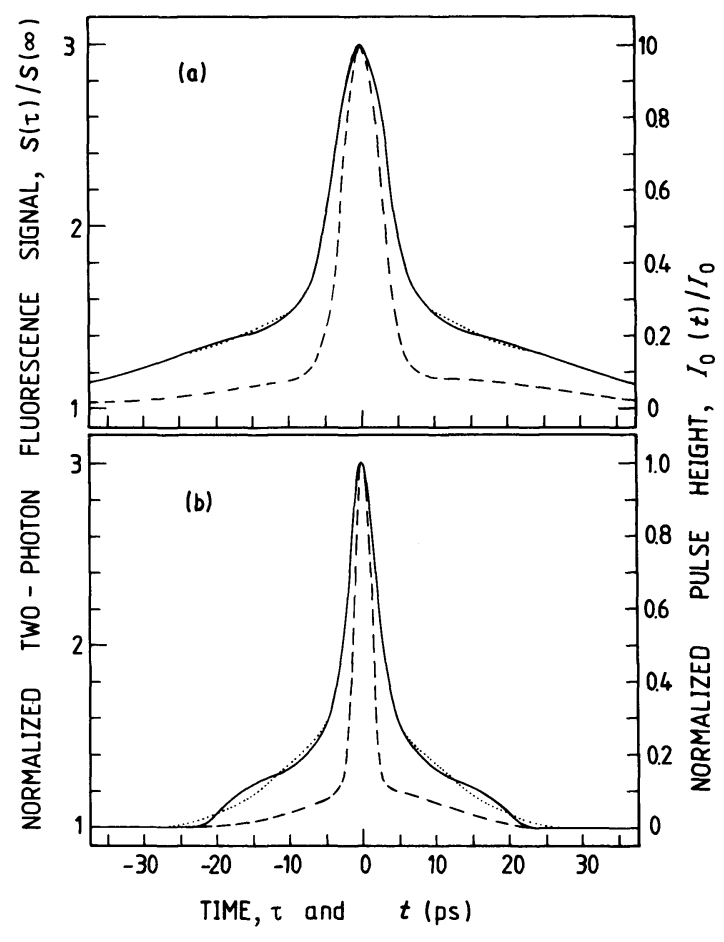

Figure 7 Two-photon fluorescence traces and pulse shapes of intracavity compressed ruby laser pulses: (a) no external saturable absorber is used; (b) pulse is shortened with external saturable absorber (DDI, $T_{0} \simeq 0.08$ ). (-) Experimental two-photon fluorescence traces. $(---)$ Decorrelated best-fit pulse shape, ( $\cdots)$ twophoton fluorescence traces belonging to dashed pulse shapes.

$(T \simeq 0.08)$. The curves in Fig. 8 belong to a passively mode-locked Nd-glass laser. The pulse compression of the self-phase modulated pulses was achieved by insertion of a birefringent filter into the laser oscillator [6]. The solid curve in Fig. 8a belongs to a pulse without external saturable absorber, whereas the solid curve in Fig. $8 \mathrm{~b}$ belongs to a pulse with external absorber $(T \simeq 0.04)$. For the ruby laser and the Nd-glass laser, rhodamine $6 \mathrm{G}$ dissolved in ethanol was used as the two-photon absorbing dye.

A comparison of the shape of the experimental two-photon fluorescence traces with calculated traces of Figs 2, 4 and 5 indicates that the experimental pulses are not sine modulated. The depicted fluorescence traces of ruby laser pulses indicate the composition of a short and a long pulse that are almost centred (Fig. 2a or b). The shown two-photon fluorescence traces of Nd-glass laser pulses are best described by two side pulses and a central spike (Fig. 4). Two-photon fluorescence traces similar to Fig. 4 are also found for intracavity compressed ruby laser pulses.

Using the computer program for calculating the two-photon fluorescence traces of combined pulses, the approximate pulse shape can be easily decorrelated from the two-photon fluorescence traces, because most pulse parameters can be directly read from the shape of the two-photon fluorescence trace. The width of the central spike gives the short pulse duration $\Delta t_{\mathrm{s}}\left(\Delta t_{\mathrm{s}}=\Delta \tau_{\mathrm{s}} / \gamma\right)$; the width of the inner side peak gives the long pulse duration $\Delta t_{1}\left(\Delta t_{1}=\left(\Delta \tau_{1}^{2}-\Delta \tau_{\mathrm{s}}^{2}\right)^{1 / 2}\right)$; the separation of the central spike from the inner side peak is equal to the pulse separation $\left(t_{\mathrm{p}}=\tau_{\mathrm{p}}\right.$, $t_{\mathrm{p} 1}=t_{\mathrm{p} 2}$ is assumed in the analysis). The height of the side pulses and the central pulse are found by an iterative loop in the computer program, where the fluorescence signal $S(0), S\left(t_{\mathrm{p}}\right)$ and $S\left(2 t_{\mathrm{p}}\right)$ are used to fit $r_{\mathrm{s}}, r_{11}$ and $r_{12}$. The pulse shape of the individual pulses is assumed to be gaussian.

The decorrelated pulse shapes are shown in Figs 7 and 8 by the dashed lines. The exact two-photon fluorescence traces to the approximate pulse shapes are included by dotted lines. The fitting is reasonably good and lies within the experimental accuracy of the two-photon-fluorescence trace measurement. The shown two-photon fluorescence traces of the ruby laser fit best to a short pulse on a broad pedestal. $t_{\mathrm{p}}>0$ is used for physical reasons (the leading part is more strongly 


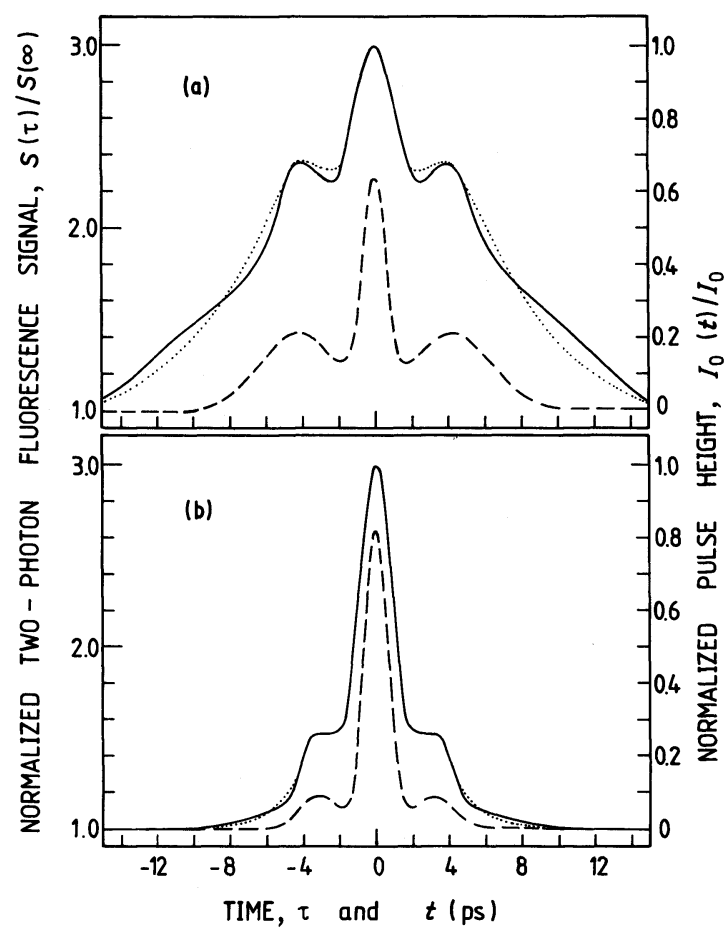

Figure 8 Two-photon fluorescence traces and pulse shapes of intracavity compressed $\mathrm{Nd}$-glass laser pulses: (a) no external saturable absorber is used; (b) pulse is shortened with external absorber (Kodak no. 9860, $\left.T_{0} \simeq 0.04\right)$. (-) Experimental two-photon fluorescence traces. (---) Decorrelated best-fit pulse shapes. $(\cdots)$ two-photon fluorescence traces belonging to dashed pulse shapes.

absorbed by mode-locking dye). The two-photon fluorescence traces presented for the Nd-glass laser are best fitted by a central pulse and two broad side pulses. The pulse shapes obtained are in good agreement with computer simulations of pulse compression of self-phase modulated pulses by the finite gain width of the active medium $[5,8]$.

\section{Conclusions}

Besides the disadvantage that from two-photon fluorescence traces the pulse shape cannot be deduced unequivocally (e.g. asymmetric pulses give symmetric fluorescence traces), the presented two-photon fluorescence trace analysis allows a good reconstruction of the shape of composed and modulated pulses. The pulses durations, temporal separation and intensity ratios of composed pulses, and the modulation depth, modulation period and overall duration of sine-modulated pulses are easily determined.

\section{Acknowledgements}

We thank Professor M. Maier for helpful discussions and Messrs J. Schmidt and F. Reil for providing the two-photon fluorescence traces belonging to the Nd-glass laser. We also thank the Deutsche Forschungsgemeinschaft for financial support and the Rechenzentrum of the University for computer time.

\section{References}

1. J. A. GIORDMAINE, P. M. RENTZEPIS, S. L. SHAPIRO and K. W. WECHT, Appl. Phys. Lett. 11 (1967) 216.

2. D. J. BRADLEY and G. H. C. NEW, Proc. IEEE 62 (1974) 313.

3. E. P. IPPEN and C. V. SHANK, in "Ultrashort Light Pulses", edited by S. L. Shapiro, Topics in Applied Physics, Vol. 18 (Springer, Berlin, 1977) p. 83.

4. H. NATHEL, D. M. GUTHALS and J. M. CLARK, 'Technical Digest of CLEO'84' (Anaheim, California, June 1984).

5. P. SPERBER and A. PENZKOFER, Opt. Commun. 54 (1985) 160.

6. J. SCHMIDT, F. REIL and A. PENZKOFER, to be published. 
7. D. VON DER LINDE, Appl. Phys. B37 (1985) 1.

8. A. PENZKOFER and F. GRAF, Opt. Quantum Electron. 17 (1985) 219.

9. A. YARIV, 'Quantum Electronics', 2nd Edn (Wiley, New York, 1975) Ch. 5.

10. R. W. MINCK, R. W. TERHUNE and C. C. WANG, Appi. Opt. 5 (1966) 1595.

11. J. C. DIELS, E. W. VAN STR YLAND and D. GOLD, in 'Picosecond Phenomena', edited by C. V. Shank, E. P. Ippen and S. L. Shapiro, Springer Series in Chemical Physics, Vol. 4 (Springer, Berlin, 1978) p. 117.

12. T. MINDL, P. HEFFERLE, S. SCHNEIDER and F. DÖRR, Appl. Phys. B31 (1983) 201.

13. J. H. BECHTEL and W. L. SMITH, J. Appl. Phys. 46 (1975) 5055.

14. D. J. BRADLEY, T. MOR ROW and M. S. PETTY, Opt. Commun. 2 (1970) 1.

15. D. J. BRAdLEY, M. H. R. hUTCHINSON, H. KOETSER, T. MORROW, G. H. NEW and M. S. PETTY, Proc. R. Soc. A328 (1972) 97.

16. S. MORY, D. LEUPOLD and R. KÖNIG, Opt. Commun. 6 (1972) 394.

17. H. J. EICHLER, D. LANGHANS and U. KLEIN, Appl. Opt. 18 (1979) 1383.

18. M. A. DUGUAY, J. W. HANSEN and S. L. SHAPIRO, IEEE J. Quantum Electron. QE-6 (1970) 725. 